\title{
Robust and Scalable Geographic Multicast Protocol for Mobile Ad Hoc Networks
}

\author{
Xiaojing Xiang* Zehua Zhou* Xin Wang $^{\dagger}$ \\ * State University of New York at Buffalo, Buffalo, NY, USA \\ $\{$ xxiang, zzhou5\}@cse.buffalo.edu \\ $\dagger$ State University of New York at Stony Brook, Stony Brook, NY, USA \\ xwang@ece.sunysb.edu
}

\begin{abstract}
Group communications are important in Mobile Ad hoc Networks (MANET). Multicast is an efficient method to implement the group communications. However, it is challenging to implement scalable, robust and efficient multicast in MANET due to the difficulty in group membership management, multicast packet forwarding and the maintenance of a tree- or mesh-based multicast structure over the dynamic topology for a large group size or network size. We propose a novel Robust and Scalable Geographic Multicast Protocol (RSGM). Scalable and efficient group membership management has been performed through zone-based structure, and the location service for group members is combined with membership management. Both the control messages and data packets are forwarded along efficient treeshape paths, but there is no need to actively maintain a tree structure, which efficiently reduces the maintenance overhead and makes the transmissions more robust to dynamics. Geographic forwarding is used to achieve further scalability and robustness. To avoid periodic flooding-based sources' announcements, an efficient source tracking mechanism is designed. Furthermore, we handle the empty zone problem faced by most zone-based routing protocols. Our simulation studies show that RSGM can scale to large group size and large network size, and a high delivery ratio is achieved by RSGM even under high dynamics.
\end{abstract}

\section{INTRODUCTION}

Group communications are important in Mobile Ad Hoc Networks (MANET). Multicast is an efficient method to realize group communications. The high dynamics of MANET, however, makes the design of routing protocols much more challenging than that of wired network. The conventional multicast protocols (e.g., [1] [2]) generally do not have good scalability due to the overhead for route searching, group membership management, and tree/mesh structure creation and maintenance over the dynamic topology of MANET.

For MANET unicast routing, geographic routing protocols (e.g., [3] [4]) have been proposed in recent years. They assume mobile nodes are aware of their own positions, and a source can obtain the destination's position through some location service (e.g., [5]). An intermediate node makes forwarding decisions based on only the destination's position and its onehop neighbors' positions learnt from periodic beaconing of the neighbors. Such local-topology based forwarding mechanism is more scalable and robust in a dynamic environment. Similarly, to achieve a scalable and robust multicasting, an option is to make use of the position information. We propose a Robust and Scalable Geographic Multicast protocol (RSGM), which can scale to a large group size and network size and is robust to network dynamics. In summary, our contributions in this work include:

- Proposing stateless distribution schemes that data packets and control messages can be sent along efficient virtualtree paths without explicitly building and maintaining a tree-structure as in general tree-based multicast protocols. This greatly reduces the control overhead and increases the reliability and scalability of the protocol.

- Making use of the position information to design a scalable and reactive zone-based scheme for efficient membership management, and a node can join and leave a group quickly.

- Supporting efficient location search of the multicast group members, by combining the location service with the membership management to avoid the need and overhead of using a separate location server.

- Introducing a home zone to track the addresses and positions of the sources, to avoid network-range periodic flooding of source information.

- Designing schemes to handle the empty-zone problems for both the member zones and home zone, which is critical in designing a zone-based protocol.

The rest of paper is organized as follows. In Section II, we discuss some related work on MANET multicast protocols. A detailed description of RSGM is given in Section III. Section IV presents the simulation studies on RSGM. Section V concludes this paper.

\section{RELATED WORK}

The conventional topology-based multicast protocols (e.g., [1] [2]) are usually composed of the following three components that generally can not scale to large network size: 1) Group membership management. The management becomes harder for a large group. 2) Creation and maintenance of a treeor mesh-based multicast structure. These will cause significant control overhead over the dynamic topology of MANET. 3) Multicast packet forwarding. The multicast packets are forwarded along the pre-built tree or mesh structure, which is vulnerable to be broken over the dynamic topology, especially in a large network with potentially longer paths. Besides these, a geographic multicast protocol also needs location service to obtain the members' positions. The geographic multicast protocols presented in [6], [7] and [8] need to put the 
information of all the group members into the packet header, which is only applicable for the small group case. Transier et al. [9] made an effort to improve the scalability of geographic multicast protocol with group size. The network terrain is divided into a quad-tree with $\mathrm{L}$ levels. The top level is the whole network and the bottom level is constructed by basic squares. A node periodically broadcasts its membership and position in basic square. And at each level of the quad-tree, every square needs to periodically flood its membership into its upper level square. Such periodical flooding are repeated for every two neighboring levels until the upmost level which is the whole network. Significant control overhead will be generated when the network size increases. With this proactive periodic membership updating scheme, a node's membership change may need to go through L levels' membership updates to make it known to the whole network, which leads to a long joining time.

\section{Robust and Scalable Geographic Multicast PROTOCOL}

RSGM supports a two-tier membership management and forwarding structure. At the lower tier, a zone structure is built based on position information and a leader is elected on demand when a zone has group members. A leader manages the group membership and collects the member nodes' positions in its zone. At the upper tier, the leaders of the member zones report the zone memberships to the sources directly along a virtual reverse-tree-based structure or through the home zone. With the knowledge of the member zones, the source forwards data packets to the zones that have group members along a virtual tree rooted at the source. After the packets arrive at the member zones, they will be further forwarded to local members through the leaders. In RSGM, we assume every node is aware of its own position (e.g., through GPS). The forwarding of data packets and most control messages follows the geographic forwarding strategy described in [3].

\section{A. Notations and Definitions}

pos: A mobile node's position coordinates (x, y).

zone: The network terrain is divided into square zones as shown in Fig. 1.

mZone (non_mZone): Member (Non_member) zone, a zone with (without) group members in it.

$z L d r$ : Zone leader.

hZone: Home zone. A zone in the network is elected as home zone to keep track of the addresses and locations of all the sources.

groupID: The address of a multicast group.

$G, S, M$ : Representing a multicast group, a source of $\mathrm{G}$ and a member of $\mathrm{G}$ respectively.

mcastTable: Multicast table. A node records the multicast information in its mcastTable. A mcastTable contains a list of group entries and hZone information (including its identification and seqNo) to be introduced later. Each group entry saves the information of a group: groupID, source list, member list and mZone list. Source list is a list of source records, which is used by group members and zLdrs to keep the sources'

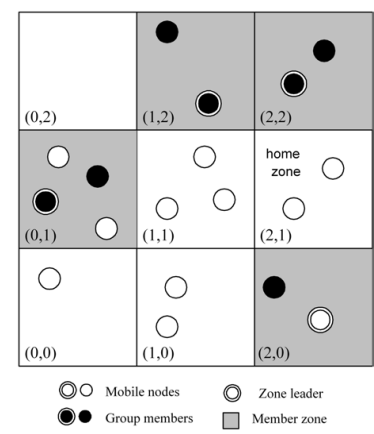

Fig. 1. Zone structure.

information. The member list is used by a zLdr to save the information of multicast group members within its local zone, and a source will record mZones in its zone list.

\section{B. Zone Construction and Maintenance}

1) Zone construction: The length of a side of the zone square is defined as zone size. Each zone is identified by a zone ID (zID). A node can calculate its $\mathrm{zID}(\mathrm{a}, \mathrm{b})$ from its pos (x, y) as: $a=\left[\frac{x-x_{0}}{z \text { zone_size }}\right]$ and $b=\left[\frac{y-y_{0}}{z \text { one_size }}\right]$, where $\left(x_{0}, y_{0}\right)$ is the position of the virtual origin. For simplicity, we assume all the zone IDs are positive. zID will also help locate a zone. In our scheme, a packet destined to a zone will be forwarded towards its center. The center position $\left(x_{c}, y_{c}\right)$ of a zone with zID (a,b) can be calculated as: $x_{c}=x_{0}+(a+0.5) \times z o n e \_s i z e$, $y_{c}=y_{0}+(b+0.5) \times$ zone_size.

2) On-demand leader election: A leader will be elected in a zone only when the zone has group members in it. When a multicast group member $\mathrm{M}$ just moves into a new zone, if the zone leader (zLdr) is unknown, $\mathrm{M}$ queries a neighbor node in the zone for zLdr. When failing to get zLdr information, M will announce itself as zLdr by flooding a LEADER message into the zone. In the case that two leaders exist in a zone, e.g., due to the slight time difference of leader queries and announcements, the one with larger ID will win as zLdr. A zLdr floods a LEADER in its zone every time interval Intval $_{\text {refresh }}$ to announce its leadership until the zone no longer has any members. If no LEADER message is received longer than $2 \times$ Intval $_{\text {refresh, }}$, a member node will wait a random period and then announce itself as zLdr when no other node announces the leadership.

\section{Group Membership Management}

1) Local group membership management: The group membership is first aggregated in the local zone. When joining or leaving a group, a member $\mathrm{M}$ sends a message REFRESH (groupIDs, pos $_{M}$ ) immediately to its zLdr to notify its membership change, where $\operatorname{pos}_{M}$ is its position and groupIDs are the addresses of the groups that $\mathrm{M}$ is a member. $\mathrm{M}$ also needs to unicast a REFRESH message to its zLdr every time interval Intval $_{\text {refresh }}$ to update its position and membership information. And a member record will be removed by the zLdr if not refreshed for longer than $2 \times$ Intval $_{\text {refresh }}$.

When $\mathrm{M}$ moves to a new zone, its next periodic REFRESH will be sent to the zLdr in the new zone. It will announce itself 


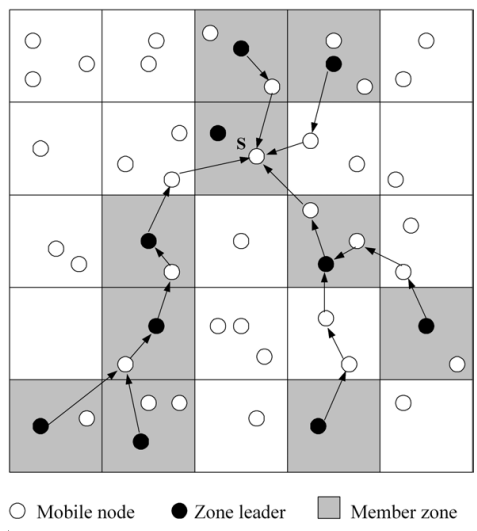

Fig. 2. The aggregation of REPORT messages.

as zLdr if the new zone has no zLdr. The moving node will still receive the multicast data packets from the old zone before its information is timed out at the old zLdr, which reduces the packet loss during the moving. For a zLdr, if its distance to the zone border is shorter than a distance threshold and the zone is still a member zone, it will handover its leadership by unicasting a LEADER to the neighbor node in its zone which is closest to the zone center. The LEADER message will continue being forwarded towards zone center until reaching a node which has no neighbor closer to the zone center than itself, and the node will take over the leadership and flood a LEADER within the zone to announce its leadership.

2) Membership management at network range: After the membership information is aggregated in the local zone, a source only needs to track the member zones (mZones).

a) Zone membership reporting by zone leaders

When a zone changes from mZone to non_mZone of $G$ or vice versa, $z$ Ldr sends a REPORT immediately to $S$ to notify the change. zLdr can get S's address and $\operatorname{pos}_{S}$ using methods described in Section III-D. A zLdr needs to send REPORT every time interval Intval ${ }_{\text {zone }}$ to $\mathrm{S}$ to refresh its zone membership information. $\mathrm{S}$ will remove a mZone record if not refreshed longer than $2 \times$ Intval $_{z o n e}$.

b) Empty zone handling

A zone may become empty when all the nodes move away. When a mZone of $\mathrm{G}$ is becoming empty, the moving out $\mathrm{zLdr}$ will notify $\mathrm{S}$ immediately to stop sending packets to the empty zone. If the moving out zLdr fails to notify $\mathrm{S}$ (e.g., zLdr suddenly dies), the packet forwarded to the empty zone will finally be dropped without being able to be delivered. The node which drops the packet will notify $\mathrm{S}$ to delete the zone from its zone list. A false deletion will be corrected when $S$ receives the periodic membership reporting from the corresponding zone.

c) Message aggregation

As compared to local messages, the control messages sent at network tier would generally traverse a longer path. We consider a reverse-tree-based aggregation scheme (Fig. 2), with which all the control messages sent towards the same destination (e.g., the source $S$ ) will be aggregated to further reduce control overhead. Different from other tree-based multicast protocols, no explicit tree-structure needs to be maintained, which avoids the overhead and improves the robustness.
Specifically, the periodic REPORT messages sent to the source can be aggregated. To facilitate the message aggregation, $\mathrm{S}$ schedules the periodic REPORT sending for the mZones. $\mathrm{S}$ inserts the next reporting time $t$ into the data packets sent out. The zLdr of a mZone schedules its next periodic REPORT to $\mathrm{S}$ at the time $t+\Delta t$, where $\Delta t$ is inversely proportional to its distance to $\mathrm{S}$. The zLdrs will form an upstream and downstream relationship according to their distances to $\mathrm{S}$. Generally the zLdrs farther away from $S$ have a shorter $\triangle t$ and will send the REPORTs earlier than the upstream zLdrs, but strict timing is not needed. When a REPORT message reaches a mZone, it is forwarded to zLdr first. When an upstream zLdr receives REPORTs from downstream zLdrs, if it hasn't sent out its REPORT, it will aggregate these REPORTs with its own REPORT, and send out the REPORT at its scheduled time. As a result, the forwarding of the REPORT messages follows a tree structure as shown in Fig. 2.

\section{Session Initialization and Source Tracking}

1) Session initiation: A multicast session $(G)$ is initiated and terminated by a source (S). To start a multicast session, $\mathrm{S}$ floods an ANNOUNCE (S, pos ${ }_{S}$, groupIDs) into the network (for reliability, promiscuous broadcasting is used in the flooding), where groupIDs are IDs of the groups (including $\mathrm{G})$ that $\mathrm{S}$ is the source. Upon receiving this message, a node (N) interested in being the group member of $G$ starts the joining process by unicasting to its zLdr a REFRESH with S's information. After session begins, $\mathrm{S}$ can piggyback its position $\left(\operatorname{pos}_{S}\right)$ to the multicast packets sent out to refresh its position at the receivers. When a member $\mathrm{M}$ moves to a new zone, the new zLdr can get S's address and $\operatorname{pos}_{S}$ from M. To terminate $\mathrm{G}, \mathrm{S}$ floods an ANNOUNCE with $\mathrm{G}$ removed from groupIDs.

2) Source tracking: A source may move during the session time. The forwarders and receivers of multicast packets from $\mathrm{S}$ can get $\operatorname{pos}_{S}$ piggy-backed in the packets, while other nodes must resort to explicit source location update mechanism to get $\operatorname{pos}_{S}$. To facilitate source location tracking and avoid networkrange periodic flooding of source information, a home zone (hZone) is used in RSGM.

Initially there is no hZone in the network. When $\mathrm{S}$ is about to announce its source role and its mcastTable has no hZone record, it will announce its current zone as hZone by inserting its zone ID (zID) and seqNo of hZone in the ANNOUNCE to be flooded into the network, where seqNo is initialized as zero. Later sources will share the elected hZone and all the nodes in hZone will maintain the sources' addresses and zIDs. Whenever a source moves to a new zone, it unicasts a REGISTER $\left(z I D_{n e w}\right)$ to hZone. The first hZone node receiving the message floods the message into hZone so that all the hZone nodes learn which zone the source is currently located in. A node just moving into hZone will get the sources' information by querying its neighbors in hZone. During the zone membership reporting (Section III-C.2), a zLdr will send REPORT to hZone if it doesn't know S's address or the source address maintained is outdated. The hZone node receiving the REPORT will forward the message towards the zone where $\mathrm{S}$ is located in. When the message arrives at S's zone, it will 
be forwarded to $\mathrm{S}$ through its zLdr. The zLdr has the position of $\mathrm{S}$ as $\mathrm{S}$ is a member of $\mathrm{G}$ and needs to send REFRESH periodically to its zLdr.

If the hZone is becoming empty, the last leaving node will announce its entering zone as the new hZone to the network, and flood into the new hZone its source list which contains the sources' information. The seqNo of hZone is increased by one every time the hZone changes. Some nodes may have no hZone information or hold an old hZone zID due to their failing to receive the hZone announcement. To handle the first case, a non-source node can get the hZone information by an expanded ring searching. For the second case, seqNo can help a node to identify the newest hZone. A message sent to hZone (e.g., REGISTER message) will carry the seqNo. A forwarding node will update its hZone information if the seqNo is larger than that it has; otherwise, it forwards this message to its recorded hZone and sends back the updated hZone information to the sending node. The seqNo will also help elect a hZone when multiple hZones exist. This can happen if a source does not know or cannot reach hZone, and announces its own zone as hZone again. The hZone with larger seqNo wins or the one with larger zID wins when having the same seqNo, and the holder of invalid hZone will be notified as described above.

\section{E. Multicast Packet Delivery}

With the membership management, the mZones are recorded by the source $\mathrm{S}$ and the local group members and their positions are recorded by zLdrs. The multicast packets are first delivered by $\mathrm{S}$ to mZones towards their zone centers. $\mathrm{S}$ sends each multicast packet to all the mZones, and to the member nodes in its own zone through zLdr. For each destination, it decides the next hop by using the geographic forwarding strategy ([3]). After all the next hops are decided, $\mathrm{S}$ unicasts to each next hop a copy of the packet which carries the list of destinations that must be reached through this hop. So the packets are forwarded along a tree-shape path although there is no need to build the tree. For robust transmissions, geographic unicast is used in packet forwarding. The packets can also be sent through broadcast to further reduce forwarding bandwidth, at the cost of reliability. When an intermediate node receives the packet, if its zone ID is not in the destination list, it will take similar action as $\mathrm{S}$ to continue forwarding the packet. If its zone is in the list, it will replace its zID in the destination list with the local members if it is a $z \mathrm{Ldr}$, or replace the zID with its zLdr otherwise, and then aggregate the sending according to the destination list as $\mathrm{S}$.

\section{Performance Evaluation}

\section{A. Simulation Overview}

We implemented RSGM within the Global Mobile Simulation (GloMoSim) [10] library. We implemented the geographic unicast forwarding strategy described in [3] with the beaconing interval set as 4s. We set RSGM's Intval refresh $_{\text {as } 4 \mathrm{~s} \text { and }}$ Intval $_{\text {zone }}$ as $6 \mathrm{~s}$. The zone size was set as $400 \mathrm{~m}$ according to our experience. For performance reference, we choose to compare with the classic mesh-based, on-demand topologybased multicast protocol ODMRP [2], and geographic multicast protocol SPBM [9].

The simulations were run with 400 nodes randomly distributed in the area of $2400 \mathrm{~m} \times 2400 \mathrm{~m}$. One multicast group was simulated with 100 group members and one source. We set the network size and group size to relatively large values to study the scalability of the protocols. The nodes moved following the random waypoint mobility model [11]. The minimum moving speed was set as $1 \mathrm{~m} / \mathrm{s}$. IEEE $802.11 \mathrm{~b}$ was used as the MAC layer protocol and the nominal transmission range was $250 \mathrm{~m}$. Each simulation lasted 500 simulation seconds. Each source sends $\mathrm{CBR}$ data packets at $8 \mathrm{Kbps}$ with packet length 512 bytes. The CBR flows start at around 30s so that the group membership management has time to initialize and stop at 480s. A simulation result was gained by averaging over six runs with different seeds.

The following metrics were studied:

1) Packet delivery ratio: The ratio of the number of packets received and the number of packets expected to be received. So the ratio is the total number of received packets over the multiplication of the group size and the number of originated packets.

2) Normalized control overhead: The total number of control message transmissions divided by the total number of received data packets.

3) Average path length: The average number of hops traversed by each delivered data packet.

4) Joining delay: The average time interval between a member joining a group and its first receiving of the data packet from that group. To obtain the joining delay, the simulations were rerun with the same settings except that all the members joined the group after the source began sending data packets.

\section{B. Simulation Results}

We evaluate the protocol performance by varying maximum moving speed from $5 \mathrm{~m} / \mathrm{s}$ to $40 \mathrm{~m} / \mathrm{s}$. From Fig. 3, under almost all the mobility cases, RSGM performs much better than ODMRP and SPBM. In all the mobility cases, the geographic multicast protocols RSGM and SPBM have higher delivery ratio as geographic forwarding is more robust to the network dynamics and both protocols use geographic unicast in their data packet transmissions to enhance reliability. RSGM has the highest delivery ratio under all the dynamics due to its robustness. The delivery ratios of ODMRP and SPBM decrease as mobility increases. Although the mesh structure used in ODMRP is more robust than general tree structure, the mesh structure is built through some kind of back learning, which is easier to become invalid due to the node movements. In SPBM, when the mobility increases, its proactive multi-level membership update mechanism can not catch the membership changes of squares in time, which leads to the decrease of delivery ratio.

In Fig. 3(b), SPBM is seen to have a significantly higher overhead than the other two protocols due to its use of periodic local-range and network-range flooding in its membership 
(a)

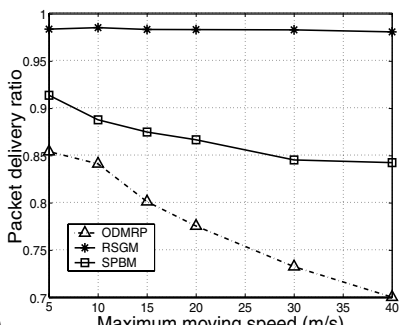

(b)

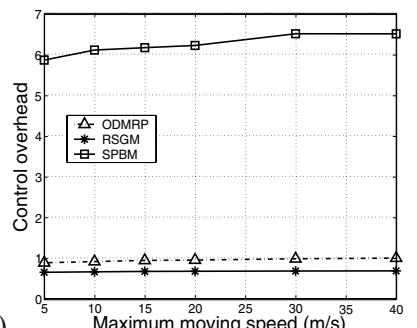

Maximum moving speed $(\mathrm{m} / \mathrm{s})^{35}$

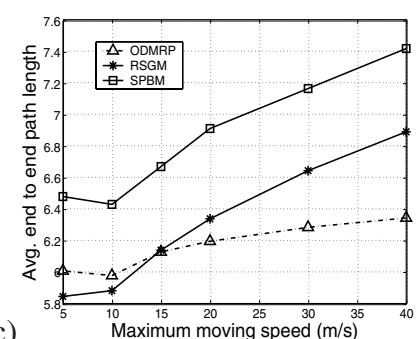

(c)

Maximum moving speed $(\mathrm{m} / \mathrm{s})$

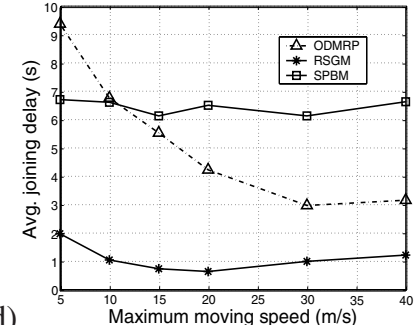

(d)

Maximum moving speed $(\mathrm{m} / \mathrm{s})$

Fig. 3. Performance vs. maximum moving speed (1 group, 1 source, 100 group members): (a) packet delivery ratio; (b) normalized control overhead; (c) average path length; (d) average joining delay.

management. The control overhead of RSGM is seen to be the lowest. There is no periodic network-range flooding in RSGM, which greatly improves the efficiency and scalability of the protocol. The control overheads of all the protocols increase as mobility increases. In RSGM, when the moving speed increases, there are more frequent zLdr changes and zone crossings, which will trigger more handover processes.

The delivery path will become non-optimal sooner under a higher dynamic environment (Fig. 3 (c)). The impact of mobility is more obvious for the path lengths of the geographic multicast protocols RSGM and SPBM. One reason is that the underlying geographic forwarding relies on periodic beaconing to refresh the neighbors' positions. With higher moving speed, such proactive beaconing cannot catch the neighbors' position changes in a timely manner and hence results in non-optimal forwarding decisions and longer routing paths as analyzed in our another work [12]. Another reason is that with a higher moving speed, the pre-built paths in ODMRP are more easily broken, so more packets with longer paths fail to reach destinations resulting in a shorter average end-to-end path for ODMRP. Between the two geographic multicast protocols, RSGM has a shorter path length. In RSGM, the packet forwarding from the source to a member zone follows the shortest path and a detour is only introduced in the destination zone by forwarding packets to group members through zLdr. In SPBM, the multicast packet forwarding follows its quadtree structure and detours happen at multiple tree levels.

In RSGM, when a node wants to join a group, it will start the joining process immediately, and the nodes can join the multicast group very fast as shown in Fig. 3(d). SPBM is seen to have the largest joining delay most of the time with the reason presented in Section II. In ODMRP, the mesh structure is built on the source's demand, and a source sends out a JOIN QUERY periodically to refresh the mesh structure. If the nodes want to join a group, they need to wait until the next mesh refreshing period. From the figure, the average joining delay of ODMRP decreases with the increase of mobility, as the higher moving speed helps a member to connect to the source more quickly in the non-geographic mesh structure.

\section{Conclusions}

We have designed a robust, scalable and efficient geographic multicast protocol RSGM for MANET in this paper. In RSGM, both the data packets and control messages will be transmitted along efficient tree-shape paths without the need of explicitly creating and maintaining a tree structure. Scalable membership management is achieved through a zone structure. A home zone is defined to provide location and address service for the sources to avoid the periodic network-range flooding of source information, and the location service for group members is combined with the membership management to avoid the overhead of searching for addresses and positions of all group members through an outside location server. The position information is used in RSGM to guide the zone structure building, membership management and packet forwarding, which reduces the maintenance overhead and leads to more robust multicast forwarding upon the topology changes. We also handle the empty zone problem which is challenging for the zone-based protocols. Our simulation results show that our protocol not only outperforms the existing geographic multicast protocol and conventional multicast protocol but can also scale to a large group size and large network size. Specifically, our protocol is more robust to network dynamics.

\section{REFERENCES}

[1] E. M. Royer and C. E. Perkins, "Multicast operation of the ad hoc ondemand distance vector routing protocol," in ACM/IEEE MOBICOM, August 1999, pp. 207-218.

[2] M. Gerla, S. J. Lee, and W. Su, "On-demand multicast routing protocol (ODMRP) for ad hoc networks," Internet draft, draft-ietf-manet-odmrp02.txt, 2000.

[3] B. Karp and H. T. Kung, "Greedy perimeter stateless routing for wireless networks," in ACM/IEEE MOBICOM, August 2000, pp. 243-254.

[4] F. Kuhn, R. Wattenhofer, Y. Zhang, and A. Zollinger, "Geometric ad-hoc routing: of theory and practice," in Int. Symposium on the Principles of Distributed Computing (PODC), 2003.

[5] J. Li and et al, "A scalable location service for geographic ad hoc routing," in ACM/IEEE MOBICOM, 2000, pp. 120-130.

[6] S. Basagni, I. Chlamtac, and V. R. Syrotiuk, "Location aware, dependable multicast for mobile ad hoc networks," Computer Networks, vol. 36, no. 5-6, pp. 659-670, August 2001.

[7] K. Chen and K. Nahrstedt, "Effective location-guided tree construction algorithms for small group multicast in MANET," in IEEE INFOCOM, 2002, pp. 1180-1189.

[8] M. Mauve, H. Fubler, J. Widmer, and T. Lang, "Position-based multicast routing for mobile ad-hoc networks," in Poster section in ACM MOBIHOC, June 2003.

[9] M. Transier, H. Fubler, J. Widmer, M. Mauve, and W. Effelsberg, "Scalable position-based multicast for mobile ad-hoc networks," in Proc. of the First International Workshop on Broadband Wireless Multimedia: Algorithms, Architectures and Applications (BroadWim 2004), October 2004.

[10] UCLA Parallel Computing Laboratory, "Glomosim," http://pcl.cs.ucla.edu/projects/glomosim/.

[11] J. Broch, D. A. Maltz, D. B. Johnson, Y.-C. Hu, and J. G. Jetcheva, "A performance comparison of multihop wireless ad hoc network routing protocols," in ACM/IEEE MOBICOM, 1998, pp. 85-97.

[12] X. Xiang, Z. Zhou, and X. Wang, "Self-adaptive on demand geographic routing protocols for mobile ad hoc networks," in IEEE INFOCOM Minisymposium, 2007. 